\title{
Characterisation of an inflammation-related epigenetic score and its association with cognitive ability
}

Anna J. Stevenson ${ }^{1,2,3}$, Daniel L. McCartney ${ }^{1}$, Robert F. Hillary ${ }^{1}$, Archie Campbell', Stewart W. Morris ${ }^{1}$, Mairead L. Bermingham', Rosie M. Walker ${ }^{1,7}$, Kathryn L. Evans ${ }^{1,7}$, Thibaud S. Boutin ${ }^{4}$, Caroline Hayward ${ }^{4}$, Allan F. McRae ${ }^{5}$, Barry W. McColl2,3, Tara L. Spires-Jones ${ }^{2,3}$, Andrew M. McIntosh ${ }^{1,6}$, lan J. Deary ${ }^{7,8}$ and Riccardo E. Marioni ${ }^{1,7^{*}}$ (D)

\begin{abstract}
Background: Chronic systemic inflammation has been associated with incident dementia, but its association with age-related cognitive decline is less clear. The acute responses of many inflammatory biomarkers mean they may provide an unreliable picture of the chronicity of inflammation. Recently, a large-scale epigenome-wide association study identified DNA methylation correlates of C-reactive protein (CRP) - a widely used acute-phase inflammatory biomarker. DNA methylation is thought to be relatively stable in the short term, marking it as a potentially useful signature of exposure.
\end{abstract}

Methods: We utilise a DNA methylation-based score for CRP and investigate its trajectories with age, and associations with cognitive ability in comparison with serum CRP and a genetic CRP score in a longitudinal study of older adults $(n=889)$ and a large, cross-sectional cohort $(n=7028)$.

Results: We identified no homogeneous trajectories of serum CRP with age across the cohorts, whereas the epigenetic CRP score was consistently found to increase with age (standardised $\beta=0.07$ and 0.01 ) and to do so more rapidly in males compared to females. Additionally, the epigenetic CRP score had higher test-retest reliability compared to serum CRP, indicating its enhanced temporal stability. Higher serum CRP was not found to be associated with poorer cognitive ability (standardised $\beta=-0.08$ and -0.05 ); however, a consistent negative association was identified between cognitive ability and the epigenetic CRP score in both cohorts (standardised $\beta=$ -0.15 and -0.08 ).

Conclusions: An epigenetic proxy of CRP may provide a more reliable signature of chronic inflammation, allowing for more accurate stratification of individuals, and thus clearer inference of associations with incident health outcomes.

Keywords: Epigenetics, DNA methylation, C-reactive protein, Inflammation, Cognitive ability

\footnotetext{
* Correspondence: Riccardo.Marioni@ed.ac.uk

${ }^{1}$ Centre for Genomic and Experimental Medicine, Institute of Genetics and

Molecular Medicine, University of Edinburgh, Edinburgh EH4 2XU, UK

${ }^{7}$ Lothian Birth Cohorts, University of Edinburgh, Edinburgh EH8 9JZ, UK

Full list of author information is available at the end of the article
}

(c) The Author(s). 2020 Open Access This article is licensed under a Creative Commons Attribution 4.0 International License, which permits use, sharing, adaptation, distribution and reproduction in any medium or format, as long as you give appropriate credit to the original author(s) and the source, provide a link to the Creative Commons licence, and indicate if changes were made. The images or other third party material in this article are included in the article's Creative Commons licence, unless indicated otherwise in a credit line to the material. If material is not included in the article's Creative Commons licence and your intended use is not permitted by statutory regulation or exceeds the permitted use, you will need to obtain permission directly from the copyright holder. To view a copy of this licence, visit http://creativecommons.org/licenses/by/4.0/ The Creative Commons Public Domain Dedication waiver (http://creativecommons.org/publicdomain/zero/1.0/) applies to the data made available in this article, unless otherwise stated in a credit line to the data. 


\section{Background}

Cognitive impairment in older age is associated with an increased risk of morbidity and mortality, and a lower quality of life [1-3]. Given the generally ageing population, and the significant personal and public health burden of age-related cognitive decline, insight into its determinants and the factors contributing to individual differences is critical. The strongest known risk factor for cognitive decline is older age, suggesting a unique facet of the ageing process is likely implicated.

Epidemiological studies have associated ageing with a progressive shift to a chronic inflammatory state. This low-grade, typically sub-acute, elevation of peripheral pro-inflammatory mediators in the absence of overt infection is strongly associated with the susceptibility to, and progression of, many age-associated diseases and is a key risk factor for mortality [4-6]. Accumulating evidence has also implicated chronic inflammation with incident dementia, but the association with pre-morbid cognitive function is less firmly defined and has generated considerable debate [7]. Evidence from large, prospective cohort and cross-sectional studies has been largely mixed, with positive, negative, and null associations identified between serum inflammatory biomarker levels and cognitive ability [8-12].

These conflicting findings may be attributed to methodological disparity between studies, including marked differences in both the ages of participants and type of cognitive assessment batteries used. However, a key issue in research utilising inflammatory biomarkers is their typically phasic responses [4]. C-reactive protein (CRP)-an acute-phase reactant of hepatic origin-is a widely used marker of inflammation. However, by definition, the plasma concentration of acute-phase proteins deviates by $25 \%$ or more in inflammatory disorders [13]. In particular, CRP is subject to swift and considerable shifts in response to injury or acute infection. Serum levels can rapidly increase up to 1000-fold from baseline, typically resolving to basal concentrations over a period of 7-12 days [14, 15]. This poses a potential issue when utilising CRP to investigate the association between chronic inflammation and health outcomes: the biological variability of the biomarker may not be stable enough to enable reliable stratification using blood samples from individuals gathered at a single time point [16].

DNA methylation is a widely studied epigenetic mechanism involving the addition of methyl groups to the DNA molecule, typically at cytosine-phosphate-guanine (CpG) dinucleotides. These modifications are involved in the regulation of gene expression and are influenced by both genetics and the environment [17]. Though DNA methylation is dynamic across the life-course, the shortterm variability in adults is thought to be relatively stable, marking it as a potentially useful signature of exposure [18-20]. Through epigenome-wide association studies (EWAS), DNA methylation signals at individual CpG sites have been associated with various health and lifestyle factors, permitting the creation of methylationbased phenotypic predictors and signatures [21-24]. Recently, a large-scale EWAS of serum CRP in adults $(n=$ 8863 and 4111 of European and African ancestries, respectively) identified DNA methylation correlates of chronic low-grade inflammation [25]. Using 7 CpG sites from this data, an inflammation-related epigenetic risk score was recently created and applied in a developmental framework investigating inflammation and child and adolescent mental health [26].

In a longitudinal study of older adults (the Lothian Birth Cohort 1936) and a large, cross-sectional cohort (Generation Scotland), we utilise this inflammationrelated epigenetic score and characterise its relationship with serum CRP levels, a genetic CRP score, and physical health and lifestyle traits that have previously been associated with CRP. We examine the longitudinal dynamics of serum CRP and the epigenetic score, establishing the stability of each over time, and if there are multiple typical trajectories characterising latent subgroups of individuals sharing a common profile. Finally, we assess the comparative associations of each predictor with cognitive ability.

\section{Methods \\ Lothian Birth Cohort 1936}

The Lothian Birth Cohort 1936 (LBC1936) is a longitudinal study comprising individuals born in 1936, most of whom completed the Scottish Mental Survey 1947 aged around 11 years. Full details on the recruitment and assessment protocols of the study have been described previously [27, 28]. Briefly, 1091 participants were recruited to the study aged around 70 years. To date, up to three further waves of testing in older age have been completed at intervals of around 3 years at mean ages of 73, 76, and 79. At each wave, data has been collected on a wealth of health outcomes, lifestyle factors, cognition, and biological measures.

\section{Generation Scotland: the Scottish Family Health Study}

Generation Scotland (GS) is a family-based genetic epidemiology cohort sampled from the general population across Scotland. The recruitment protocol and cohort characteristics are described in detail elsewhere [29, 30]. Initially, 7953 individuals aged between 18 and 65 years were recruited between 2006 and 2011 from General Practitioner registries. Family members of these subjects aged between 18 and 99 years were then approached to participate. The final cohort comprised around 24,000 subjects. Data were collected on various cognitive, 
psychiatric, and health measures, and DNA samples were collected for genotyping and methylation profiling.

\section{Phenotype preparation C-reactive protein}

LBC1936 Serum CRP was measured from venesected whole-blood samples. Levels were quantified using both a low-sensitivity assay $(\mathrm{mg} / \mathrm{L})$ performed using a dryslide immuno-rate method on an OrthoFusion 5.1 F.S analyser (Ortho Clinical Diagnostics). This assay cannot distinguish values less than $3 \mathrm{mg} / \mathrm{L}$, thus all readings of $<3 \mathrm{mg} / \mathrm{L}$ were assigned a value of $1.5 \mathrm{mg} / \mathrm{L}$ [31].

Generation Scotland CRP was quantified at the University of Glasgow using a commercial high-sensitivity assay on an automated analyser (c311, Roche Diagnostics, UK). Manufacturer's calibration and quality control were employed. CRP data was available for 419 individuals. These samples had been selected as father/offspring pairs in a telomere length study.

\section{Cognitive ability}

LBC1936 Fluid-type cognitive ability encompasses the capacity to perform basic information processing and extemporary thinking tasks, rather than those involving acquired knowledge or experience. This cognitive domain typically exhibits a decline with age. Scores on different tests of fluid-type cognitive ability are typically highly correlated, indicative of a latent general cognitive ability factor. Here, we derived a single general fluid-type cognitive ability score $\left(g_{f}\right)$ for each participant from the first un-rotated principal component of a principal components analysis on six of the Wechsler Adult Intelligence Scale-III tests. These tests assessed four different cognitive domains: letter-number sequencing and digit span backwards (working memory), digit-symbol coding and symbol search (processing speed), matrix reasoning (non-verbal reasoning), and block design (constructional ability) [32]. This component explained 53\% of the variance across the 6 tests, with individual test loadings ranging from 0.66 to 0.78 . Full details on the testing protocol for the cognitive tests in LBC1936 have been reported previously $[27,33]$.

Generation Scotland Similarly to LBC1936, $g_{f}$ was obtained for each participant from the first un-rotated principal component of a principal components analysis of three tests of cognitive ability: logical memory, verbal fluency (executive function), and digit-symbol coding (processing speed). This component explained 50\% of the variance across the three tests with test loadings ranging from 0.63 to 0.78 . Logical memory was assessed using the Wechsler Memory Scale-III [34]. Verbal fluency and digit symbol-coding were tested using the Wechsler Adult Intelligence Scale III [32]. Additional information regarding the cognitive variables in GS has been described previously $[35,36]$.

\section{Physical and lifestyle variables}

LBC1936 The variables previously associated with CRP levels included in the analysis in LBC1936 were as follows: body mass index (BMI: the ratio of weight in kilogram divided by height in square metres), self-reported smoking status (current smoker, ex-smoker, never smoker), alcohol consumption in a typical week (units), and social deprivation. Social deprivation was measured using the Scottish Index of Multiple Deprivation (SIMD). The SIMD ranks geographical areas in Scotland based on current income, employment, health, education, skills and training, geographic access to services, housing, and crime. The SIMD provides a standardised measure of relative deprivation throughout Scotland.

Generation Scotland The variables in GS were all measured as described for LBC1936, the only exception being smoking status in which ex-smokers were divided into those who had quit within the previous 12 months of their blood sample date and those who had quit prior to that.

\section{Inflammation-related poly-epigenetic score}

Details on the DNA methylation preparation for each cohort are presented in Additional file 1. An inflammation-related poly-epigenetic score (DNAm CRP) was derived for each participant as described by Barker et al. [26]. Briefly, methylation beta values were extracted for the $7 \mathrm{CpG}$ sites shown to have the strongest evidence of a functional association with serum CRP levels. These values were multiplied by their respective regression weights (corresponding to change in DNA methylation beta values per 1 unit increase in natural log-transformed CRP) taken from the largest EWAS of CRP to-date and summed to generate a single DNAm CRP score for each participant (Additional file 2: Table S1 [25]). All of the regression weights from the EWAS were negative, resulting in a higher DNAm CRP score (i.e. closer to zero) corresponding to a prediction of increased CRP levels. Of the 7 CpG sites included in the original measure, one was not available on the EPIC array and was therefore was unavailable in the GS data (cg06126421). A DNAm CRP score inclusive of the remaining $6 \mathrm{CpG}$ sites was utilised in analyses for GS.

It should be noted that LBC1936 contributed 296 high-sensitivity CRP samples (from wave 2) to the EWAS of CRP from which the DNAm SCRP score was 
derived [25]. This may mean results from this cohort are overfitted; however, the LBC1936 individuals represent a small subset of the discovery sample $(n=8863)$, and the probes were selected to be highly significant so it is unlikely this had a significant impact on results.

\section{Genetic score for CRP}

Information on genotyping for each cohort is presented in Additional file 1. An additive weighted genetic score for CRP was constructed in both cohorts from the 18 single nucleotide polymorphisms (SNPs) that passed the genome-wide threshold $\left(p<5 \times 10^{-8}\right)$ in the largest available genome-wide association study (GWAS) of CRP to date [23]. Weighted dosages were calculated by multiplying the dose of each risk allele by the effect estimate from the GWAS (Additional file 2: Table S2). An imputation quality score of $>0.8$ was applied to the SNPs.

\section{Statistical analysis}

Pearson correlations were calculated between serum CRP, the DNAm CRP score (and its component CpGs), and the genetic CRP score in each cohort. Inter-wave correlations and intraclass correlation coefficients were estimated for the DNAm CRP score and serum CRP over the four waves of follow-up in LBC1936 to assess the stability of the measures over time.

Linear mixed models were used to investigate the change in the DNAm CRP score and serum CRP over the four waves in LBC1936. Sex was included as a fixed effect, age (years) as the timescale, and participant ID as a random effect on the intercept. Latent class mixed models were used to account for potential population heterogeneity in the trajectories and to determine the number of differing trajectories in both the serum and DNAm CRP data. Initially, a model with a single class was run. Additional classes were then added (up to 5) until the optimal number of classes was established using the Bayes Information Criterion (BIC) and the mean posterior probability of belonging to each class (mean probability $>0.65$ ).

To investigate the validity of the DNAm and genetic CRP scores as markers of inflammation, linear regression models were run with phenotypes that have previously been associated with circulating CRP levels: BMI [37], smoking [38-40], alcohol [41], and deprivation [42, 43]. Age and sex have previously been identified as strong determinants of circulating CRP levels and thus were included as covariates in these models. Each phenotype was additionally included as a covariate unless fitted as the dependent variable of interest. Linear regression models were then used to obtain the cross-sectional associations between $g_{f}$ and serum CRP, the DNAm CRP score, and the genetic CRP score. Models were adjusted for the aforementioned correlated phenotypes. Methylation set was included as an additional covariate in GS (see Additional file 1). In LBC1936, models were conducted at wave 1 (age $\sim 70$ ) of the study. To determine which CpG site in the DNAm CRP score was most relevant to the cognitive association, fully adjusted models with each of the CpGs that comprise the score as independent variables were computed. Correction for multiple testing was applied using the false discovery rate (FDR $p<0.05$ ) [44].

Linear mixed effect models, with the baseline DNAm CRP score or serum CRP included as a fixed effect interaction with age, were used to test the prediction of subsequent change in cognitive ability. Participant ID was fitted as a random effect on the intercept.

Numeric variables were scaled to have a mean of zero and a variance of 1 . Serum CRP and alcohol intake data were log-transformed (natural log) prior to analyses due to positive skews in their distribution.

Statistical analysis was performed in $\mathrm{R}$ version 3.5.0 [45]. Linear mixed-effect models and latent class mixed models were implemented using the 'ImerTest' and the 'lcmm' package, respectively [46, 47].

\section{Results}

\section{Cohort information}

Descriptive statistics of all the variables used in analyses are presented in Table 1. LBC1936 is an older cohort than GS (LBC1936 wave 1: mean $=69.5$ years; GS: mean $=50.9$ years), with a more even balance between sexes (LBC1936: 49\% female; GS: 58\% female). LBC1936 had a higher mean CRP $(5.26 \mathrm{mg} / \mathrm{L})$ compared to GS $(2.65$ $\mathrm{mg} / \mathrm{L})$. The mean genetic score for CRP was 2.16 in LBC1936 and 2.15 in GS.

\section{Correlation between serum CRP, DNAm CRP score, and genetic score}

In both cohorts, the Pearson correlations between the DNAm CRP score and serum $\log (\mathrm{CRP})$ were moderate (LBC1936: $r=0.34,95 \%$ CI [0.28, 0.4]; GS: $r=0.28$ $[0.19,0.36])$, with the DNAm CRP score showing a stronger correlation with serum CRP than any one of its composite $\mathrm{CpGs}$ in both cohorts (Additional file 3. Figures S1 and S2). In LBC1936, the correlation between the genetic score for CRP and serum CRP was 0.21 $[0.15,0.27]$, and in GS, it was 0.29 [0.2, 0.38]. The correlation between the DNAm CRP score and the genetic score was low in LBC1936 ( $r=0.07[0.06,0.14])$ and not significantly different to zero in GS $(r=-0.01[-0.03$, 0.016]). 
Table 1 Cohort characteristics

\begin{tabular}{|c|c|c|c|c|c|}
\hline & $n$ & Mean & Median & Range & SD \\
\hline LBC1936 wave 1 & 1091 & - & - & - & - \\
\hline Age (years) & - & 69.53 & 69.54 & $67.61,71.29$ & 0.83 \\
\hline Sex (\% female) & 49.8 & - & - & - & - \\
\hline $\mathrm{CRP}(\mathrm{mg} / \mathrm{L})$ & 1054 & 5.26 & 3.0 & $1.5,90$ & 6.78 \\
\hline DNAm CRP & 894 & - & -0.019 & $-0.024,-0.015$ & 0.0013 \\
\hline Genetic score & 1005 & 2.16 & 2.16 & $1.23,2.83$ & 0.25 \\
\hline$g_{f}$ & 1070 & 0.096 & 0.12 & $-3.43,3.017$ & 1.0091 \\
\hline $\mathrm{BMI}\left(\mathrm{kg} / \mathrm{m}^{2}\right)$ & 1083 & 27.78 & 27.39 & $16.023,48.52$ & 4.36 \\
\hline $\begin{array}{l}\text { Alcohol (units/ } \\
\text { week) }\end{array}$ & 1063 & 9.93 & 6 & 0,70 & 12.12 \\
\hline Smoking status & 894 & - & - & - & - \\
\hline Current & 102 & - & - & - & - \\
\hline Ex & 375 & - & - & - & - \\
\hline Never & 417 & - & - & - & - \\
\hline SIMD & 1083 & 5342 & 5342 & 4,6505 & 1907 \\
\hline Generation Scotland & 7028 & - & & & - \\
\hline Age (years) & - & 50.89 & 52.75 & $18,93.3$ & 12.96 \\
\hline Sex (\% female) & 58.2 & - & - & - & - \\
\hline CRP (ng/mL) & 419 & 2.65 & 1.21 & $0.15,63.25$ & 5.59 \\
\hline DNAm CRP & 7028 & $\begin{array}{l}- \\
0.012\end{array}$ & -0.013 & $\begin{array}{l}-0.016,- \\
0.0089\end{array}$ & $\begin{array}{l}< \\
0.001\end{array}$ \\
\hline Genetic score & 7028 & 2.15 & 2.17 & $1.14,2.91$ & 0.26 \\
\hline$g_{f}$ & 6880 & 0.0063 & 0.042 & $-4.15,3.89$ & 0.99 \\
\hline $\mathrm{BMI}\left(\mathrm{kg} / \mathrm{m}^{2}\right)$ & 6988 & 26.93 & 26.15 & $14.78,51.29$ & 5.089 \\
\hline $\begin{array}{l}\text { Alcohol (units/ } \\
\text { week) }\end{array}$ & 6414 & 10.49 & 8 & 0,73 & 11.017 \\
\hline Smoking status & 6854 & - & - & - & - \\
\hline Current & 1135 & - & - & - & - \\
\hline Ex & 2167 & - & - & - & - \\
\hline Never & 3552 & - & - & - & - \\
\hline SIMD & 6693 & 3953 & 4456 & 3,6505 & 1857 \\
\hline
\end{tabular}

LBC1936 Lothian Birth Cohort 1936, CRP C-reactive protein, DNAm DNA methylation, $g_{f}$ general cognitive ability score, SIMD Scottish Index of Multiple Deprivation, $B M I$ body mass index

\section{Trajectories of the DNAm CRP score and serum CRP}

Plots of the observed trajectories of both serum $\log (\mathrm{CRP})$ and the DNAm CRP score in LBC1936 are shown in Fig. 1a. The observed trajectories of serum CRP in LBC1936 have been reported previously [48]. $\log (\mathrm{CRP})$ was found to decline over the 9 years of follow-up $(\beta=-0.014, \mathrm{SE}=0.005, p=$ 0.003). Conversely, the DNAm CRP score was found to increase by an average of $0.07 \mathrm{SD}$ per year $(\mathrm{SE}=0.004, p<2 \times$ $10^{-16}$ ). An interaction was identified between age and sex, indicating that the DNAm CRP score inclined faster over time in males compared to females $(\beta=0.018, \mathrm{SE}=0.007, p=$ 0.009). Plots of the pseudo-trajectories of serum $\log (\mathrm{CRP})$ and the DNAm CRP score in GS are shown in Fig. 1c. Both, $\log (\mathrm{CRP})$ and the DNAm CRP score increased with age $(\beta=$ $0.011, \mathrm{SE}=0.004, p=0.008 ; \beta=0.013, \mathrm{SE}=0.0008, p<2 \times$ $10^{-16}$, respectively). Again, an interaction between age and sex was identified in the DNAm CRP score, with a more rapid rise in males than females $(\beta=0.017, \mathrm{SE}=0.002, p=2$ $\left.\times 10^{-16}\right)$.

To further investigate possible population heterogeneity in the trajectories, latent class analyses were conducted in LBC1936. The model with two latent classes had the best fit for serum $\log (\mathrm{CRP})$ (Additional file 2: Table S3). Though the BIC was minimised with four latent classes, this included a class of only 27 individuals and a low mean posterior class membership probability for class 1 (65.6\%). The model with three classes also exhibited a low posterior probability of belonging to each latent class for class 2 (61.4\%); therefore, the model with two classes was selected in which the mean probabilities for class membership were high -0.95 and 0.86 for class 1 and class 2, respectively. The two underlying mean trajectories are presented in Fig. 1b. In this model, class $1(n=811,75.5 \%)$ exhibited a stable trajectory, with no evident change over the eighth decade $(\beta=-0.0025, \mathrm{SE}=0.005, p=0.96)$. Class $2(n=$ $263,24.5 \%)$ showed a decline over time, likely indicating a subset of individuals exhibiting regression to the mean $(\beta$ $\left.=-0.032, \mathrm{SE}=0.008, p=1 \times 10^{-4}\right)$. For the DNAm CRP score, class determination indicated the model with one class best fit the data. Here, a small elevation over time was identified $\left(\beta=0.06, \mathrm{SE}=0.005, p<1 \times 10^{-5}\right)$.

\section{Stability of the DNAm CRP score and serum CRP}

The intraclass correlation coefficient for serum CRP over the four waves of follow-up in LBC1936 was 0.72 (95\% CI $\left.[0.69,0.74], p<2 \times 10^{-16}\right)$. For the DNAm CRP score, it was $0.82\left([0.75,0.86], p<2 \times 10^{-16}\right)$, ranging from 0.60 (cg27023597) to $0.94(\mathrm{cg} 06126421)$ in the 7 CpG sites that comprised the score. The correlations of the DNAm CRP score and serum CRP between each of the four waves in LBC1936 are presented in Additional file 3: Figure S3. The inter-wave correlations of serum $\log (\mathrm{CRP})$ ranged from 0.3 (wave 1 -wave 4 ) to 0.45 (wave 1 -wave 2). The correlations of the DNAm CRP score between waves were stronger, ranging from 0.53 (wave 1 -wave 4) to 0.75 (wave 2 -wave 3 ), indicating an enhanced temporal stability.

\section{Associations with determinants of CRP}

The associations between serum CRP, the DNAm CRP score, and the health and lifestyle phenotypes are presented in Table 2. No associations were found between the genetic score and any of the phenotypes in either cohort (Additional file 2: Table S4). Higher serum CRP was found to associate with a higher BMI and smoking status in both cohorts (LBC1936 BMI: $\beta=0.27, \mathrm{SE}=$ $0.03, p_{\mathrm{FDR}}=2.1 \times 10^{-17} ;$ smoking: $\log$ odds $=0.30, \mathrm{SE}=$ 

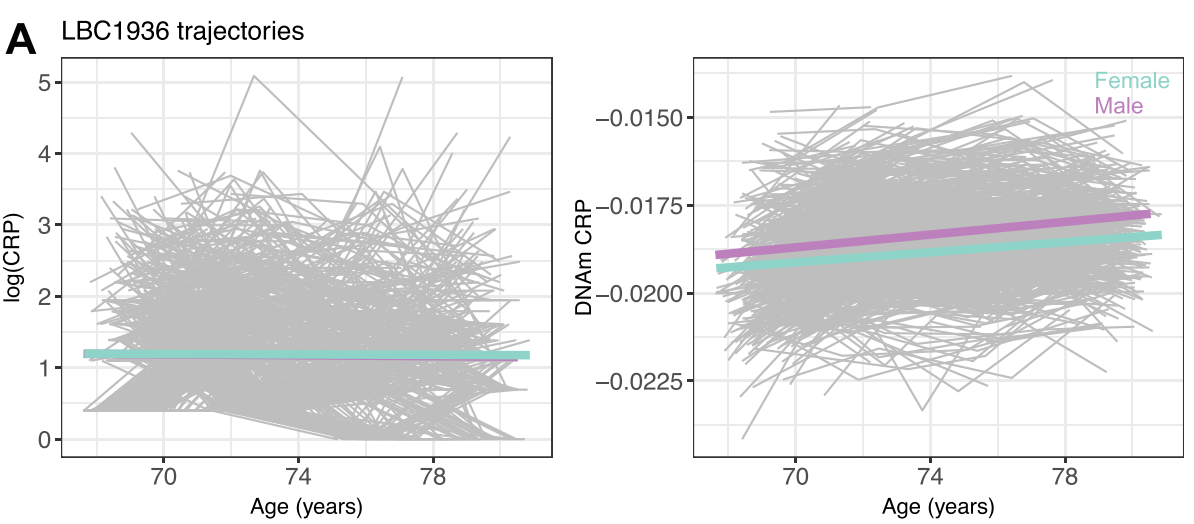

B LBC1936 latent trajectories
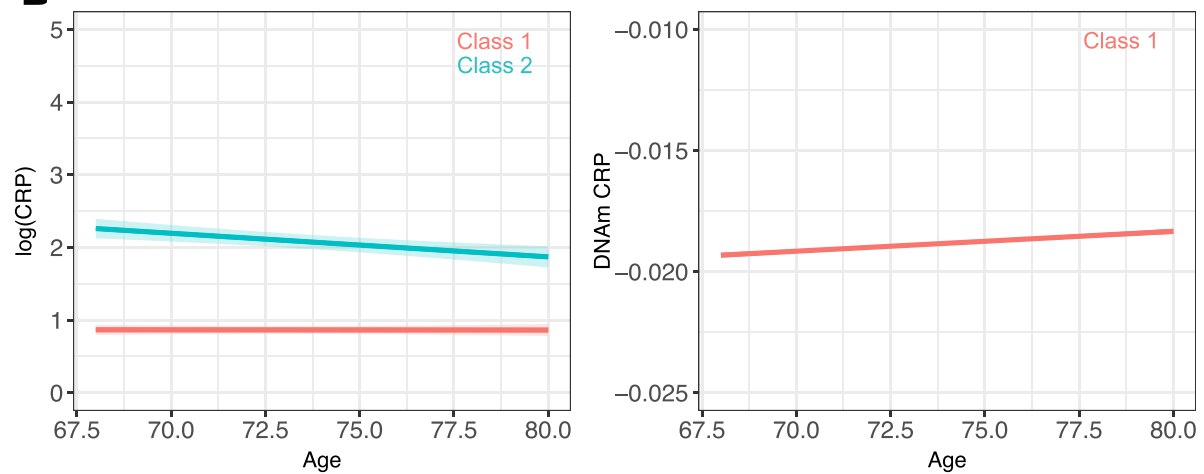

C GS pseudo-trajectories
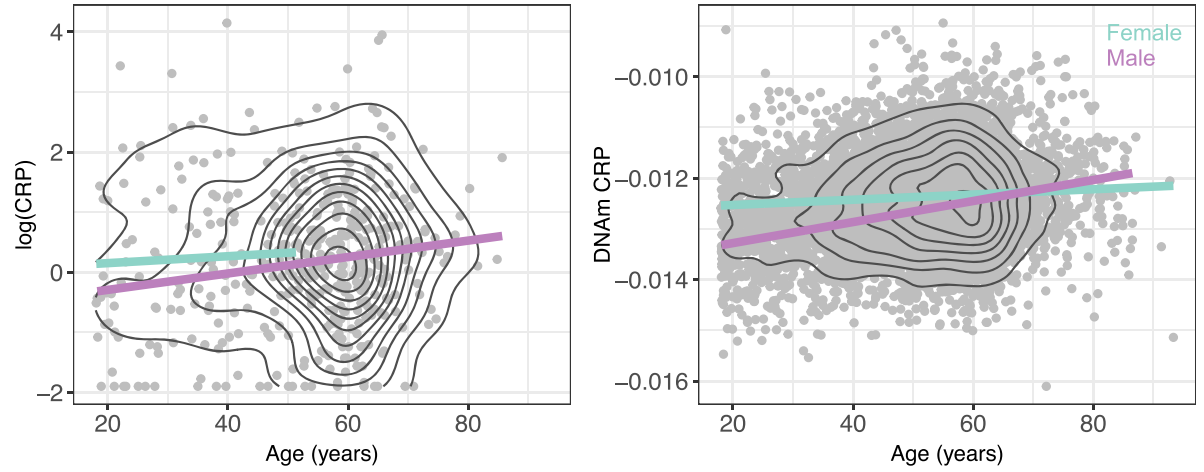

Fig. 1 Trajectories of serum CRP and the DNAm CRP score over age. a Observed trajectories in the Lothian Birth Cohort 1936. Grey lines represent individual participant trajectories with regression lines shown for females and males in blue and purple, respectively. $\mathbf{b}$ Latent trajectories in Lothian Birth Cohort 1936. Latent classes are shown in red (class 1) and blue (class 2). c Pseudo-trajectories in Generation Scotland for CRP $(n=419)$ and the DNAm CRP score $(n=7028)$. Regression lines are as in (a). Black lines represent density. DNAm = DNA methylation; $\mathrm{CRP}=\mathrm{C}$-reactive protein

$0.07, p_{\mathrm{FDR}}=1.4 \times 10^{-5} ; \mathrm{GS}$ BMI: $\beta=0.29, \mathrm{SE}=0.04$, $p_{\mathrm{FDR}}=3.3 \times 10^{-10}$; smoking: $\log$ odds $=0.26, \mathrm{SE}=0.11$, $\left.p_{\mathrm{FDR}}=0.03\right)$ but not with alcohol intake or social deprivation $(p \geq 0.14)$. In the subset of individuals with measured CRP in GS, the DNAm CRP score associated with BMI $\left(\beta=0.15, \mathrm{SE}=0.06, p_{\mathrm{FDR}}=0.019\right)$ and alcohol intake $\left(\beta=0.19, \mathrm{SE}=0.06, p_{\mathrm{FDR}}=0.003\right)$ but not with smoking $(p=0.29)$. In LBC1936 and the full GS cohort, a higher DNAm CRP score robustly associated with a higher
BMI (LBC1936: $\beta=0.14, \mathrm{SE}=0.04, p_{\mathrm{FDR}}=2.4 \times 10^{-4}$; GS: $\left.\beta=0.19, \mathrm{SE}=0.01, p_{\mathrm{FDR}}=2.4 \times 10^{-38}\right)$, increased alcohol intake (LBC1936: $\beta=0.12, \mathrm{SE}=0.03, p_{\mathrm{FDR}}=0.001$; GS: $\beta=0.15$, SE $\left.=0.01,9.6 \times 10^{-24}\right)$, a more deprived socioeconomic status (LBC1936: $\beta=-0.12$, $\mathrm{SE}=0.03, p_{\mathrm{FDR}}=7.2 \times 10^{-4}$; GS: $\beta=-0.07, \mathrm{SE}=0.01$, $\left.p_{\text {FDR }}=1.4 \times 10^{-6}\right)$, and being a smoker $($ LBC1936: log odds $=0.54, \mathrm{SE}=0.07, p_{\mathrm{FDR}}=1.5 \times 10^{-12}$; GS: $\log$ odds $=$ $\left.0.24, \mathrm{SE}=0.03, p_{\mathrm{FDR}}=1.5 \times 10^{-18}\right)$. 
Table 2 Associations between physical and lifestyle traits, and serum CRP and the DNAm CRP score

\begin{tabular}{|c|c|c|c|c|c|c|c|c|c|c|c|c|}
\hline \multicolumn{13}{|c|}{ Lothian Birth Cohort 1936} \\
\hline \multirow[t]{2}{*}{ CRP } & & & & & \multicolumn{8}{|c|}{ DNAm CRP } \\
\hline & $\beta$ & SE & P & FDR $P$ & \multicolumn{2}{|l|}{$\beta$} & \multicolumn{3}{|l|}{ SE } & \multicolumn{2}{|l|}{$P$} & FDR $P$ \\
\hline BMI & 0.27 & 0.03 & $3.1 \times 10^{-18}$ & $2.1 \times 10^{-17}$ & \multicolumn{2}{|l|}{0.14} & \multicolumn{3}{|l|}{0.04} & \multicolumn{2}{|c|}{$8.1 \times 10^{-5}$} & $2.4 \times 10^{-4}$ \\
\hline Alcohol & -0.004 & 0.03 & 0.26 & 0.41 & \multicolumn{2}{|l|}{0.12} & \multicolumn{3}{|l|}{0.03} & \multicolumn{2}{|c|}{$5.9 \times 10^{-4}$} & 0.001 \\
\hline SIMD & -0.05 & 0.03 & 0.14 & 0.25 & \multicolumn{2}{|l|}{-0.12} & \multicolumn{3}{|l|}{0.03} & \multicolumn{2}{|c|}{$2.7 \times 10^{-4}$} & $7.2 \times 10^{-4}$ \\
\hline Smoking & 0.30 & 0.07 & $4.2 \times 10^{-6}$ & $1.4 \times 10^{-5}$ & \multicolumn{2}{|l|}{0.54} & \multicolumn{2}{|l|}{0.07} & & \multicolumn{2}{|c|}{$2.7 \times 10^{-13}$} & $1.5 \times 10^{-12}$ \\
\hline \multicolumn{13}{|c|}{ Generation Scotland } \\
\hline \multirow[t]{2}{*}{ CRP } & & & & & \multicolumn{4}{|c|}{ DNAm—subset with measured CRP } & \multicolumn{4}{|c|}{ DNAm—full dataset } \\
\hline & $\beta$ & SE & $P$ & FDR $P$ & $\beta$ & SE & $P$ & FDR P & $\beta$ & SE & $P$ & FDR $P$ \\
\hline BMI & 0.29 & 0.04 & $7.2 \times 10^{-11}$ & $3.3 \times 10^{-10}$ & 0.15 & 0.06 & 0.009 & 0.019 & 0.19 & 0.01 & $8.7 \times 10^{-40}$ & $2.4 \times 10^{-38}$ \\
\hline Alcohol & 0.01 & 0.07 & 0.83 & 0.91 & 0.19 & 0.06 & 0.001 & 0.003 & 0.15 & 0.01 & $7.1 \times 10^{-25}$ & $9.6 \times 10^{-24}$ \\
\hline SIMD & 0.03 & 0.05 & 0.53 & 0.64 & -0.04 & 0.06 & 0.45 & 0.64 & -0.07 & 0.01 & $3.7 \times 10^{-7}$ & $1.4 \times 10^{-6}$ \\
\hline Smoking & 0.26 & 0.11 & 0.01 & 0.03 & 0.16 & 0.11 & 0.17 & 0.29 & 0.24 & 0.03 & $1.7 \times \times 10^{-19}$ & $1.5 \times 10^{-18}$ \\
\hline
\end{tabular}

Log odds are presented for smoking. Analysis was run at wave 1 of the Lothian Birth Cohort $1936(n=1091)$. Significant associations are highlighted in bold

SIMD Scottish Index of Multiple Deprivation, BMI body mass index, CRP C-reactive protein, DNAm DNA methylation, SE standard error, FDR false discovery rate

\section{Associations with cognitive ability}

The associations between the individual predictors-serum CRP, the DNAm CRP score, and the genetic score-and $g_{f}$ are presented in Table 3. CRP has previously been found to associate with cognitive ability cross-sectionally at wave 1 of LBC1936 [49]. In fully adjusted models in LBC1936, neither serum CRP nor the genetic CRP score was associated with $g_{f}\left(p_{\mathrm{FDR}} \geq 0.16\right)$, but an inverse association was identified with the DNAm CRP score $\left(\beta=-0.08, \mathrm{SE}=0.03, p_{\mathrm{FDR}}\right.$ $=0.04)$. Similar results were found in GS, with no association with serum CRP or the genetic score (Table $3, p_{\text {FDR }} \geq$ 0.71 ) but a higher DNAm CRP score associated with poorer cognitive ability $\left(\beta=-0.04, \mathrm{SE}=0.01, p_{\mathrm{FDR}}=0.04\right)$. The association between $g_{f}$ and each of the CpG sites within the DNAm CRP score is presented in Additional file 2: Table S5. In both cohorts, cg18181703 (SOCS3) was associated with $g_{f}$ with a larger effect size than that of the DNAm CRP

Table 3 Associations between $g_{f}$ and individual predictors

\begin{tabular}{lllll}
\hline & Standardised $\beta$ & SE & Raw $P$ & FDR $P$ \\
\hline Lothian Birth Cohort 1936 & -0.033 & 0.03 & 0.26 & 0.41 \\
$\log ($ CRP) & 0.052 & 0.03 & 0.08 & 0.16 \\
Genetic score & -0.084 & 0.03 & $\mathbf{0 . 0 1}$ & $\mathbf{0 . 0 4}$ \\
DNAm CRP & & & & \\
Generation Scotland & & 0.05 & 0.74 & 0.77 \\
log(CRP) & -0.016 & 0.01 & 0.57 & 0.71 \\
Genetic score & 0.007 & 0.01 & $\mathbf{0 . 0 1}$ & $\mathbf{0 . 0 4}$ \\
DNAm CRP & -0.035 & &
\end{tabular}

Significant associations are highlighted in bold. Analysis was run at wave 1 of LBC1936 $(n=1091)$

CRP C-reactive protein, DNAm DNA methylation, SE standard error, FDR false discovery rate score, suggesting this locus is particularly important in driving the observed cognitive associations.

The longitudinal associations between baseline serum CRP and DNAm CRP score and $g_{f}$ in LBC1936 are presented in Additional file 2: Table S6. There was no evidence to suggest either CRP or the DNAm CRP score at wave 1 of LBC1936 was predictive of subsequent change in $g_{f}$ over the 4 years of follow-up $(p \geq 0.69)$.

\section{Discussion}

We identified discrepant trajectories of serum CRP with age across two cohorts, whereas the DNAm CRP score was invariably found to increase with age and to do so more rapidly in males than in females. The DNAm CRP score additionally showed robust associations with health and lifestyle variables previously found to impact CRP levels. We found that a higher DNAm CRP score associated with lower cognitive function; however, neither baseline serum CRP nor the DNAm CRP score was associated with longitudinal change in cognitive ability.

Whereas ageing is considered to be linked to systemically raised inflammation [4], we identified divergent dynamics of serum CRP in the assessed cohorts, with both increasing and declining trajectories seen as a function of age. Contrastingly, we identified congruous increases in the DNAm CRP score in relation to age. In LBC1936, the increase per year was greater than that seen in GS, likely due to the inclusion of only older individuals within the cohort who may be more likely to experience a progressive elevation in inflammation $[4,50]$. Furthermore, we consistently found a significant interaction between the DNAm CRP score and sex, with males having a steeper incline compared to females. This is conceivably due to men having a lower life- 
expectancy, and thus accelerated immune dysregulation, captured by the inflammation-related epigenetic score. Latent class analysis suggested the majority of individuals in LBC1936 exhibited stable dynamics of serum CRP over the eighth decade, with a slight decline identified in a smaller subgroup. The decline is likely due to a regression to the mean and highlights the acute nature and serum CRP and the challenges of utilising it as a biomarker of chronic systemic inflammation in studies where it is quantified only once, or even at multiple time-points with large sampling intervals across the life-course of a longitudinal study. Contrastingly, the analysis of the DNAm CRP score indicated a consistent population trajectory that increased over time. Moreover, the DNAm CRP score had higher inter-wave correlations and test-retest coefficients and more robust associations with the determinants of serum CRP, indicating it had less associated error than phenotypic CRP. As DNAm is considered relatively stable, the DNAm CRP score could conceivably be regarded as a cumulative, composite measure of inflammation akin to the HbA1c test typically utilised in diabetic patients to obtain a 3-month average blood glucose recording [51]. The DNAm CRP score may then have the potential to provide a more sensitive biomarker of chronic inflammation, overcoming the noise that the phasic nature of serum CRP introduces, allowing for more reliable analyses of chronic inflammatory variance and its associative relationships.

In both cohorts, we reported an inverse association between cognitive ability and the DNAm CRP score, but no association with the genetic score or measured CRP. It seems this association is largely driven by cg18181703 (SOCS3). This site has previously been associated with higher scores on tests of vocabulary and speed of information processing in an EWAS meta-analysis of cognitive abilities (LBC1936 contributed to this study [52]), as well as with type 2 diabetes and BMI-both strong correlates of circulating CRP levels [53, 54]. SOCS3 itself is involved in regulating pro-inflammatory cytokine signalling, and its expression has been found to be upregulated in the brains of patients with Alzheimer's disease, suggesting it may be an important site in linking peripheral metabolic and inflammatory processes with central pathology and function [55]. Our results are consistent with a recent study identifying a negative association between the DNAm CRP score at birth and cognitive function in early life [26]. This, coupled with our results from GS, whose participant ages span early-adulthood to later-life, suggests inflammation and cognition may be related across the life-course rather than necessarily exclusively in older age. The larger effect size in LBC1936, however, suggests the association becomes more pronounced in older adults. Neither serum CRP nor the DNAm CRP score were found to associate with longitudinal change in cognitive ability over time indicating no predictive relationship between either measure of inflammation and cognitive decline. Previous studies have suggested that inflammation in middle age, rather than later life, may be a more powerful determinant of age-related cognitive change, and it would be interesting to utilise the DNAm CRP score in a longitudinal cohort spanning this age range to examine this hypothesis further $[12,56]$.

The strengths of this study include the large sample sizes and the range of longitudinal data available. We demonstrate that the DNAm CRP score could provide a proxy measure when CRP itself is not quantified, allowing for the investigation of inflammation in cohorts with only methylation data available. Limitations include the typically healthy nature of the two cohorts meaning our findings may not extrapolate to a more general population. Additionally, serum CRP is not directly produced by immune cells, and thus, it is, in itself, a proxy marker of inflammation. A chronic signature of inflammation might also manifest in whole blood through changes in cell type proportions which should also be considered in future analyses. While the DNAm CRP score may be capturing a more reliable picture of inflammation than measured CRP, it would be desirable to investigate its relationship with a panel of inflammatory mediators and to create epigenetic scores of other inflammatory biomarkers to test their comparative performance. Finally, no causal analysis was conducted in this study, so it remains to be determined (a) if CRP has a direct effect on methylation or indeed the opposite is true, though this has begun to be addressed elsewhere $[25,57]$; and (b) if inflammation-related DNAm is causal of poorer cognition, vice-versa, or both are influenced by a confounding factor.

\section{Conclusion}

Research into the complex relationship between systemic inflammation and cognitive ability relies upon accurate characterisation of inflammatory mediators to enable reliable conclusions. Here, we show that an inflammationrelated poly-epigenetic score may provide a more stable index of chronic, low-grade inflammation in comparison with serum CRP itself. We found the epigenetic score associated more robustly with cognitive ability compared to the measured phenotype, demonstrating the potential value in using epigenetic information in place of labile phenotypes. DNAm signatures of acute inflammatory markers may provide a better signature of chronic inflammation, allowing for more reliable stratification of individuals, and thus clearer inference of its association with incident health outcomes. 


\section{Supplementary information}

Supplementary information accompanies this paper at https://doi.org/10. 1186/s13148-020-00903-8.

Additional file 1. Supplementary methods.

Additional file 2: Table S1. CpG sites and relative weights (from Lighthart et al.) used to generate the DNAm CRP score. Table S2. SNPs and relative weights (from Dehghan et al.) used to generate the genetic score for CRP. Table S3. Summary of latent class analyses for log(CRP) and the DNAm CRP score in the Lothian Birth Cohort 1936. Table S4. Associations with determinants of CRP and the genetic CRP score. Table S5. Association between individual CPG sites and general cognitive ability $\left(g_{f}\right)$ in the Lothian Birth Cohort 1936 and Generation Scotland Table S6. Longitudinal associations between the baseline DNAm CRP score and serum CRP and general cognitive ability $\left(g_{f}\right)$ over the four waves of follow-up in the Lothian Birth Cohort 1936.

Additional file 3. Figure S1. Pearson correlations between serum CRP, the DNAm CRP score and the genetic score. Figure S2. Correlations between individual CPGs comprising the DNAm CRP score and serum CRP in the Lothian Birth Cohort 1936 and Generation Scotland. Figure S3. Inter-wave Pearson correlations DNAm CRP score and serum CRP in Lothian Birth Cohort 1936

\section{Abbreviations}

CRP: C-reactive protein; CpG: Cytosine-phosphate-guanine;

EWAS: Epigenome-wide association study; LBC1936: Lothian Birth Cohort

1936; GS: Generation Scotland; gf: General fluid-type cognitive ability; SIMD: Scottish Index of Multiple Deprivation; DNAm: DNA methylation; SNP: Single nucleotide polymorphism; GWAS: Genome-wide association study; FDR: False discovery rate

\section{Acknowledgements}

The authors thank all individuals and project team members who have contributed to both GS and to the 'STRADL: Stratifying Resilience and Depression Longitudinally' follow-up study. GS received core support from the Chief Scientist Office of the Scottish Government Health Directorates (CZD/16/6) and the Scottish Funding Council (HR03006). The authors thank all LBC1936 study participants and research team members who have contributed, and continue to contribute, to ongoing studies.

\section{Authors' contributions}

Conception and design: AJS and REM. Data analysis: AJS, DLM, and REM. Drafting the article: AJS and REM. Data preparation: DLM, RFH, RMW, MB, SWM, TSB, CH, AFM, and REM. Data collection: AMM, KLE, and IJD. Revision of the article: all authors. All authors read and approved the final manuscript.

\section{Funding}

Genotyping and DNA methylation profiling of the GS samples was carried out by the Genetics Core Laboratory at the Wellcome Trust Clinical Research Facility, Edinburgh, Scotland, and was funded by the Medical Research Council UK and the Wellcome Trust (Wellcome Trust Strategic Award 'STratifying Resilience and Depression Longitudinally' (STRADL) reference 104036/Z/14/Z). LBC1936 is supported by Age UK (Disconnected Mind program) and the Medical Research Council (MR/M01311/1). Methylation typing was supported by Centre for Cognitive Ageing and Cognitive Epidemiology (Pilot Fund award), Age UK, The Wellcome Trust Institutional Strategic Support Fund, The University of Edinburgh, and The University of Queensland.

This work was in part conducted in the Centre for Cognitive Ageing and Cognitive Epidemiology, which is supported by the Medical Research Council and Biotechnology and Biological Sciences Research Council (MR/ K026992/1) and which supports IJD. AJS and RFH are supported by funding from the Wellcome Trust 4-year PhD in Translational Neuroscience-training the next generation of basic neuroscientists to embrace clinical research [108890/Z/15/Z]. REM and DLMCC are supported by Alzheimer's Research UK major project grant ARUK-PG2017B-10. TSJ and BWM are supported by the UK Dementia Research Institute which receives its funding from DRI Ltd., funded by the UK Medical Research Council, Alzheimer's Society, and Alzheimer's Research UK. TSJ is additionally supported by the European Research
Council (ERC) under the European Union's Horizon 2020 research and innovation programme under grant agreement no. 681181

\section{Availability of data and materials}

LBC1936 data are not publicly available due to them containing information that could compromise participant consent and confidentiality. LBC1936 data are available on request from the Lothian Birth Cohort Study, Centre for Cognitive Ageing and Cognitive Epidemiology, University of Edinburgh. According to the terms of consent for Generation Scotland participants access to data must be reviewed by the Generation Scotland Access Committee. Applications should be made to access@generationscotland.org.

\section{Ethics approval and consent to participate}

All components of GS received ethical approval from the NHS Tayside Committee on Medical Research Ethics (REC reference number: 05/S1401/89). GS has also been granted Research Tissue Bank status by the Tayside Committee on Medical Research Ethics (REC reference number: 10/S1402/20), providing generic ethical approval for a wide range of uses within medical research.

Ethical permission for the LBC1936 was obtained from the Multi-Centre Research Ethics Committee for Scotland (MREC/01/0/56) and the Lothian Research Ethics Committee (LREC/2003/2/29). Written informed consent was obtained from all participants.

\section{Consent for publication}

Not applicable

\section{Competing interests}

The authors declare that they have no competing interests.

\section{Author details}

${ }^{1}$ Centre for Genomic and Experimental Medicine, Institute of Genetics and Molecular Medicine, University of Edinburgh, Edinburgh EH4 2XU, UK. ${ }^{2}$ UK Dementia Research Institute, Edinburgh Medical School, University of Edinburgh, Edinburgh, UK. ${ }^{3}$ Centre for Discovery Brain Sciences, University of Edinburgh, Edinburgh, UK. ${ }^{4}$ Medical Research Council Human Genetics Unit, Institute of Genetics and Molecular Medicine, Western General Hospital, University of Edinburgh, Edinburgh EH4 2XU, UK. ${ }^{5}$ Institute for Molecular Bioscience, University of Queensland, Brisbane QLD, Australia. ${ }^{6}$ Division of Psychiatry, Royal Edinburgh Hospital, University of Edinburgh, Edinburgh EH10 5HF, UK. 'Lothian Birth Cohorts, University of Edinburgh, Edinburgh EH8 9JZ, UK. ${ }^{8}$ Department of Psychology, University of Edinburgh, Edinburgh EH8 9JZ, UK

Received: 25 March 2020 Accepted: 9 July 2020

Published online: 27 July 2020

\section{References}

1. Batty GD, Deary IJ, Zaninotto P. Association of cognitive function with cause-specific mortality in middle and older age: follow-up of participants in the English longitudinal study of ageing. American journal of epidemiology. 2016;183(3):183-90.

2. Singh-Manoux A, Sabia S, Kivimaki M, Shipley MJ, Ferrie JE, Marmot MG. Cognition and incident coronary heart disease in late midlife: the Whitehall II study. Intelligence. 2009;37(6):529-34.

3. Deary IJ, Corley J, Gow AJ, Harris SE, Houlihan LM, Marioni RE, et al. Ageassociated cognitive decline. British medical bulletin. 2009;92:135-52.

4. Franceschi C, Campisi J. Chronic inflammation (inflammaging) and its potential contribution to age-associated diseases. The journals of gerontology Series A, Biological sciences and medical sciences. 2014; 69(Suppl 1):S4-9.

5. Coussens LM, Werb Z. Inflammation and cancer. Nature. 2002;420(6917): 860-7.

6. Bonaccio M, Di Castelnuovo A, Pounis G, De Curtis A, Costanzo S, Persichillo $\mathrm{M}$, et al. A score of low-grade inflammation and risk of mortality: prospective findings from the Moli-sani study. Haematologica. 2016;101(11): 1434-41.

7. Bettcher BM, Kramer JH. Longitudinal inflammation, cognitive decline, and Alzheimer's disease: a mini-review. Clinical pharmacology and therapeutics. 2014;96(4):464-9. 
8. Engelhart MJ, Geerlings MI, Meijer J, Kiliaan A, Ruitenberg A, van Swieten JC, et al. Inflammatory proteins in plasma and the risk of dementia: the rotterdam study. Archives of neurology. 2004;61(5):668-72.

9. Koyama A, O'Brien J, Weuve J, Blacker D, Metti AL, Yaffe K. The role of peripheral inflammatory markers in dementia and Alzheimer's disease: a meta-analysis. The journals of gerontology Series A, Biological sciences and medical sciences. 2013;68(4):433-40.

10. Buchman AS, Bennett DA. High CRP with "normal" cognition: a resilient phenotype in old age. Neurology. 2012;79(11):1078-9.

11. Zheng F, Xie W. High-sensitivity C-reactive protein and cognitive decline: the English Longitudinal Study of Ageing. Psychological medicine. 2018; 48(8):1381-9.

12. Walker KA, Gottesman RF, Wu A, Knopman DS, Gross AL, Mosley TH Jr, et al. Systemic inflammation during midlife and cognitive change over 20 years: the ARIC Study. Neurology. 2019;92(11):e1256-e67.

13. Morley JJ, Kushner I. Serum C-reactive protein levels in disease. Annals of the New York Academy of Sciences. 1982;389:406-18.

14. Gabay C, Kushner I. Acute-phase proteins and other systemic responses to inflammation. The New England journal of medicine. 1999;340(6):448-54.

15. Sproston NR, Ashworth JJ. Role of C-reactive protein at sites of inflammation and infection. Front Immunol. 2018;9:754

16. Koenig W, Sund M, Frohlich M, Lowel H, Hutchinson WL, Pepys MB. Refinement of the association of serum C-reactive protein concentration and coronary heart disease risk by correction for within-subject variation over time: the MONICA Augsburg studies, 1984 and 1987. American journal of epidemiology. 2003;158(4):357-64.

17. Jaenisch R, Bird A. Epigenetic regulation of gene expression: how the genome integrates intrinsic and environmental signals. Nature genetics. 2003;33(Suppl):245-54

18. Dolinoy DC, Weidman JR, Jirtle RL. Epigenetic gene regulation: linking early developmental environment to adult disease. Reproductive toxicology (Elmsford, NY). 2007;23(3):297-307.

19. Byun HM, Nordio F, Coull BA, Tarantini L, Hou L, Bonzini M, et al. Temporal stability of epigenetic markers: sequence characteristics and predictors of short-term DNA methylation variations. PloS one. 2012;7(6):e39220.

20. Talens RP, Boomsma DI, Tobi EW, Kremer D, Jukema JW, Willemsen G, et al. Variation, patterns, and temporal stability of DNA methylation: considerations for epigenetic epidemiology. The FASEB Journal. 2010;24(9): 3135-44.

21. McCartney DL, Hillary RF, Stevenson AJ, Ritchie SJ, Walker RM, Zhang Q, et al. Epigenetic prediction of complex traits and death. Genome biology. 2018;19(1):136

22. Hamilton OKL, Zhang Q, McRae AF, Walker RM, Morris SW, Redmond P, et al. An epigenetic score for BMI based on DNA methylation correlates with poor physical health and major disease in the Lothian Birth Cohort. International Journal of Obesity. 2019

23. Sayols-Baixeras S, Subirana I, Lluis-Ganella C, Civeira F, Roquer J, Do AN, et al. Identification and validation of seven new loci showing differential DNA methylation related to serum lipid profile: an epigenome-wide approach. The REGICOR study. Human molecular genetics. 2016;25(20):4556-65.

24. Guay SP, Voisin G, Brisson D, Munger J, Lamarche B, Gaudet D, et al. Epigenome-wide analysis in familial hypercholesterolemia identified new loci associated with high-density lipoprotein cholesterol concentration. Epigenomics. 2012;4(6):623-39.

25. Ligthart S, Marzi C, Aslibekyan S, Mendelson MM, Conneely KN, Tanaka T, et al. DNA methylation signatures of chronic low-grade inflammation are associated with complex diseases. Genome biology. 2016;17(1):255.

26. Barker ED, Cecil CAM, Walton E, Houtepen LC, O'Connor TG, Danese A, et al. Inflammation-related epigenetic risk and child and adolescent mental health: a prospective study from pregnancy to middle adolescence. Development and psychopathology. 2018;30(3):1145-56.

27. Deary IJ, Gow AJ, Pattie A, Starr JM. Cohort profile: the Lothian Birth Cohorts of 1921 and 1936. International journal of epidemiology. 2012;41(6):1576-84.

28. Taylor AM, Pattie A, Deary IJ. Cohort profile update: the Lothian Birth Cohorts of 1921 and 1936. International journal of epidemiology. 2018;47(4): 1042-r.

29. Smith BH, Campbell H, Blackwood D, Connell J, Connor M, Deary IJ, et al. Generation Scotland: the Scottish Family Health Study; a new resource for researching genes and heritability. BMC medical genetics. 2006;7:74.

30. Smith BH, Campbell A, Linksted P, Fitzpatrick B, Jackson C, Kerr SM, et al. Cohort profile: Generation Scotland: Scottish Family Health Study (GS:SFHS).
The study, its participants and their potential for genetic research on health and illness. International journal of epidemiology. 2013;42(3):689-700.

31. Aribisala BS, Wiseman S, Morris Z, Valdes-Hernandez MC, Royle NA, Maniega SM, et al. Circulating inflammatory markers are associated with magnetic resonance imaging-visible perivascular spaces but not directly with white matter hyperintensities. Stroke. 2014;45(2):605-7.

32. Wechsler D. Wechsler Adult Intelligence Scale - Third Edition (WAIS-III); 1997

33. Deary IJ, Gow AJ, Taylor MD, Corley J, Brett C, Wilson V, et al. The Lothian Birth Cohort 1936: a study to examine influences on cognitive ageing from age 11 to age 70 and beyond. BMC geriatrics. 2007;7:28.

34. Wechsler D. Wechsler Memory Scale - Third Edition. 1997.

35. Marioni RE, Campbell A, Scotland G, Hayward C, Porteous DJ, Deary IJ. Differential effects of the APOE e4 allele on different domains of cognitive ability across the life-course. European journal of human genetics : EJHG. 2016;24(6):919-23.

36. Hagenaars SP, Harris SE, Clarke TK, Hall L, Luciano M, Fernandez-Pujals AM, et al. Polygenic risk for coronary artery disease is associated with cognitive ability in older adults. International journal of epidemiology. 2016.

37. Kao TW, Lu IS, Liao KC, Lai HY, Loh CH, Kuo HK. Associations between body mass index and serum levels of C-reactive protein. South African medical journal =. Suid-Afrikaanse tydskrif vir geneeskunde. 2009;99(5):326-30.

38. Wannamethee SG, Lowe GD, Shaper AG, Rumley A, Lennon L, Whincup PH. Associations between cigarette smoking, pipe/cigar smoking, and smoking cessation, and haemostatic and inflammatory markers for cardiovascular disease. European heart journal. 2005;26(17):1765-73.

39. Shiels MS, Katki HA, Freedman ND, Purdue MP, Wentzensen N, Trabert B, et al. Cigarette smoking and variations in systemic immune and inflammation markers, JNCl: Journal of the National Cancer Institute. 2014; 106(11).

40. Ryu SY, Lee YS, Park J, Kang MG, Kim KS. Relations of plasma high-sensitivity C-reactive protein to various cardiovascular risk factors. J Korean Med Sci. 2005;20(3):379-83

41. Oliveira A, Rodriguez-Artalejo F, Lopes C. Alcohol intake and systemic markers of inflammation--shape of the association according to sex and body mass index. Alcohol and alcoholism (Oxford, Oxfordshire). 2010;45(2): 119-25.

42. Nazmi A, Victora CG. Socioeconomic and racial/ethnic differentials of Creactive protein levels: a systematic review of population-based studies. BMC Public Health. 2007;7(1):212.

43. Koster A, Bosma H, Penninx BWJH, Newman AB, Harris TB, van Eijk JTM, et al. Association of inflammatory markers with socioeconomic status. The Journals of Gerontology: Series A. 2006;61(3):284-90.

44. Benjamini $Y$, Hochberg $Y$. Controlling the false discovery rate: a practical and powerful approach to multiple testing. Journal of the Royal statistical society: series B (Methodological). 1995;57(1):289-300.

45. Revelle W. psych: Procedures for psychological, psychometric, and personality research, Northwestern University, Evanston, Illinois, USA, https:// CRAN.R-project.org/package $=$ psych Version $=1.8 .12 .2018$.

46. Proust-Lima C, Philipps V, Diakite A, Liquet B, Proust MC. Package 'Icmm'. 2019

47. Kuznetsova A, Brockhoff PB, Christensen RHB. ImerTest Package: tests in linear mixed effects models. 2017. 2017;82(13):26.

48. Stevenson AJ, McCartney DL, Harris SE, Taylor AM, Redmond P, Starr JM et al. Trajectories of inflammatory biomarkers over the eighth decade and their associations with immune cell profiles and epigenetic ageing. Clinical Epigenetics. 2018;10(1):159.

49. Luciano M, Marioni RE, Gow AJ, Starr JM, Deary IJ. Reverse causation in the association between C-reactive protein and fibrinogen levels and cognitive abilities in an aging sample. Psychosomatic medicine. 2009;71(4):404-9.

50. Sanada F, Taniyama Y, Muratsu J, Otsu R, Shimizu H, Rakugi H, et al. Source of chronic inflammation in aging. Front Cardiovasc Med. 2018;5:12.

51. Gonen B, Rubenstein A, Rochman H, Tanega SP, Horwitz DL. Haemoglobin A1: an indicator of the metabolic control of diabetic patients. Lancet (London, England). 1977;2(8041):734-7.

52. Marioni RE, MCRae AF, Bressler J, Colicino E, Hannon E, Li S, et al. Metaanalysis of epigenome-wide association studies of cognitive abilities. Mol Psychiatry. 2018;23(11):2133-44.

53. Ali O, Cerjak D, Kent JW, James R, Blangero J, Carless MA, et al. Methylation of SOCS3 is inversely associated with metabolic syndrome in an epigenome-wide association study of obesity. Epigenetics. 2016; 11(9):699-707. 
54. Chambers JC, Loh M, Lehne B, Drong A, Kriebel J, Motta V, et al. Epigenome-wide association of DNA methylation markers in peripheral blood from Indian Asians and Europeans with incident type 2 diabetes: a nested case-control study. The lancet Diabetes \& endocrinology. 2015;3(7): 526-34.

55. Walker DG, Whetzel AM, Lue LF. Expression of suppressor of cytokine signaling genes in human elderly and Alzheimer's disease brains and human microglia. Neuroscience. 2015;302:121-37.

56. Laurin D, David Curb J, Masaki KH, White LR, Launer L. Midlife C-reactive protein and risk of cognitive decline: a 31-year follow-up. Neurobiology of Aging. 2009;30(11):1724-7.

57. Walton E, Hemani G, Dehghan A, Relton C, Smith GD. Systematic evaluation of the causal relationship between DNA methylation and C-reactive protein. bioRxiv. 2018:397836.

\section{Publisher's Note}

Springer Nature remains neutral with regard to jurisdictional claims in published maps and institutional affiliations.

Ready to submit your research? Choose BMC and benefit from:

- fast, convenient online submission

- thorough peer review by experienced researchers in your field

- rapid publication on acceptance

- support for research data, including large and complex data types

- gold Open Access which fosters wider collaboration and increased citations

- maximum visibility for your research: over $100 \mathrm{M}$ website views per year

At BMC, research is always in progress.

Learn more biomedcentral.com/submissions 